\title{
Combined melatonin-adipose derived mesenchymal stem cell therapy effectively protected the testis from testicular torsion-induced ischemia- reperfusion injury
}

\section{Yen-Ta Chen}

Chang Gung Memorial Hospital Kaohsiung Branch

\section{Fei-Chi Chuang}

Chang Gung Memorial Hospital Kaohsiung Branch

\section{Chih-Chao Yang}

Chang Gung Memorial Hospital Kaohsiung Branch

\section{John Y. Chiang}

National Sun Yat-sen University

\section{Pei-Hsun Sung}

Chang Gung Memorial Hospital Kaohsiung Branch

\section{Yi-Ching Chu}

Chang Gung Memorial Hospital Kaohsiung Branch

\section{Chi-Ruei Huang}

Chang Gung Memorial Hospital Kaohsiung Branch

Kuan-Hui Huang

Chang Gung Memorial Hospital Kaohsiung Branch

Hon-Kan Yip ( $\nabla$ han.gung@msa.hinet.net)

Chang Gung Memorial Hospital Kaohsiung Branch https://orcid.org/0000-0002-6305-5717

\section{Research}

Keywords: testis, ischemia-reperfusion injury, melatonin, adipose-derived mesenchymal stem cells

Posted Date: March 13th, 2021

DOl: https://doi.org/10.21203/rs.3.rs-297008/v1

License: (1) This work is licensed under a Creative Commons Attribution 4.0 International License. Read Full License 


\section{Abstract}

Background: This study tested the hypothesis that combined melatonin (Mel) and adipose-derived mesenchymal stem cell (ADMSC) treatment was superior to either one alone on protecting the testis against acute testicular torsion-induced ischemia-reperfusion (TTIR) injury.

Methods and Results: Male-adult SD rats $(n=30)$ were equally categorized into group 1 (sham-operated control), group 2 [TTIR/by torsion of right/left testis (i.e., ischemia) with rotated 720 degrees counterclockwisely for $2 \mathrm{~h}$, then detorsion (i.e., reperfusion) to the original position for $72 \mathrm{~h}$ ], group 3 (TTIR $+\mathrm{Mel} /$ intraperitoneal administration/50 mg/kg at 30 minutes after ischemia, followed by $20 \mathrm{mg}$ at $3 \mathrm{~h}$ and days $1 / 2 / 3$ after TTIR), group $4\left(T T I R+A D M S C / 1.2 \times 10^{6}\right.$ cells/intravenous administration at 30 minutes after ischemia, followed by days $1 / 2$ TTIR) and group 5 (TTIR + Mel + ADMSC). The result showed that the protein expressions of oxidative-stress (NOX-1/NOX-2/oxidized-protein), apoptotic/mitochondrial-damaged (mitochondrial-Bax/cleaved-caspase3/cleaved-PARP/cytosoliccytochrome C) and fibrotic (TGF-ß/Smad3) biomarkers as well as testicular damage scores were lowest in group 1, highest in group 2 and significantly higher in groups 3/4 than in group 5, but they showed no difference between groups $3 / 4$, whereas the protein expressions of androgen receptor (AR) and vimentin showed an opposite pattern of oxidative stress (all $p<0.0001$ ). The cellular levels of inflammation (MMP9/MPO/CD68) exhibited an identical pattern, whereas the numbers of Sertoli cells, a-tubulin, AR and vimentin as well as thickness of seminiferous tubule exhibited an opposite pattern of oxidative stress among the groups (all $p<0.0001$ ).

Conclusion: Mel-ADMSCs effectively protected the testis against TTIR injury.

\section{Introduction}

Testicular torsion due to twisting of the spermatic cord is a serious urological emergency that is most commonly encountered in those of newborns, children and adolescent males [1, 2]. Testicular torsion causes testicular injury, leading to potentially serious sequelae of infertility and subfertility; thus, rapid diagnosis and prompt treatment are extremely important [3-5]. Undoubtedly, the duration and degree of cord twisting are the two major contributors that are closely related to the severity of the testicular injury $[2,6]$.

It is well known that testicular ischemia-reperfusion (IR) injury represents the essential pathophysiology of testicular torsion, with ischemia caused by twisting of the spermatic cord, and reperfusion on its subsequent release. Accordingly, the successful treatment of testicular torsion is clearly identified in two distinct steps. First, to quickly release the torsion (i.e., detorsion) by surgical intervention [1-7]. This procedure which is a standard method for treatment of testicular torsion usually poses no difficulty for an interventional urologist. Second, to prevent reperfusion injury by pharmacomodulation. However, currently, no effective pharmaceutics for reducing the gonad damage from reperfusion injury due to the central pathological mechanisms of testicular injury following testicular torsion are not entirely 
understood, despite the overproduction of reactive oxygen species (ROS)/free radicals and reactive nitrogen species generated during the IR process have been implicated as two of the main factors in cellular and tissue damage [8-10]. Of particular interest is that while the ipsilateral testicular damage after spermatic cord torsion is better clarified, the status of the contralateral testis remains obscure [11$16]$.

Vast research have revealed that melatonin, a powerful free-radical scavenger and metal chelator [17] with the ability of attenuating oxidative stress [18-20] and inflammatory reactions [21, 22] as well as stabilizing cellular membranes [23], furnished protective effects against sepsis-caused kidney organ damage [24]. Additionally, rich data has demonstrated that stem cell therapies were effective and promising for many disease entities which are refractory to traditional treatment [25-28]. Of these, adipose-derived mesenchymal stem cells (ADMSCs) have been prove to augment angiogenesis and tissue regeneration, leading to improved ischemia-related organ dysfunction, as well as has antiinflammatory and immunomodulatory capacity for ameliorating IR-induced organ dysfunction [24, 2931].

Intriguingly, abundant experimental studies have brought to light that IR caused organ dysfunction and tissue damage are mainly through inflammation, generation of oxidative stress/ROS and DNA damage which are significantly inhibited by Mel or ADMSC therapy [21, 22, 24, 27, 29-31]. However, whether cell therapy would offer benefit for protecting the testis from testicular torsion-induced IR (TTIR) injury is currently unknown. Accordingly, this study tested whether combined Mel-ADMSC therapy would be superior to either one alone for protecting the testis against reperfusion injury.

\section{Materials And Methods}

\section{Ethics}

All animal procedures were approved by the Institute of Animal Care and Use Committee at Kaohsiung Chang Gung Memorial Hospital (Affidavit of Approval of Animal Use Protocol No. 2017012401) and performed in accordance with the Guide for the Care and Use of Laboratory Animals.

Animals were housed in an Association for Assessment and Accreditation of Laboratory Animal Care International (AAALAC; Frederick, MD, USA)-approved animal facility in our hospital with controlled temperature and light cycles $\left(24^{\circ} \mathrm{C}\right.$ and $12 / 12$ light cycle).

\section{The procedure and protocol of testicular torsion to induce acute TTIR injury}

The procedure and protocol were based on the previous report with some modifications [32]. In detail, pathogen-free, adult male Sprague-Dawley (SD) rats weighing 320-350 g (Charles River Technology, BioLASCO Taiwan Co. Ltd., Taiwan) were used in the present study. All animals were anesthetized by inhalational $2.0 \%$ isoflurane, placed in a supine position on a warming pad at $37^{\circ} \mathrm{C}$ for midline laparotomies. The sham-operated control (SC) group received ilioinguinal incisions only, while IR was 
induced in other groups of animals by rotating $720^{\circ}$ in a counterclockwise direction and maintained for $2 \mathrm{~h}$ by fixing the right and left testes to the scrotum with a 4-0 silk suture. Detorsion (i.e., returned to the normal position) was then performed by untwisting the testes to original position and maintaining the position.

The animals $(n=30)$ were equally categorized into group 1 (i.e., SC, only by a sham operation of the left and right testes without any IR procedure), group 2 [TTIR, i.e., by torsion of right and left testes (i.e., ischemia) with 720 degrees counterclockwise rotation for $2 \mathrm{~h}$, then detorsion (i.e., reperfusion) to the original position for 72h], group 3 [TTIR + Mel (intraperitoneal administration of Mel $50 \mathrm{mg} / \mathrm{kg}$ at 30 minutes after ischemia, followed by $20 \mathrm{mg}$ at $3 \mathrm{~h}$ and days 1, 2 and 3 after IR injury)], group 4 [TTIR + ADMSCs $\left(1.2 \times 10^{6}\right.$ cells by intravenous administration at 30 minutes after ischemia, followed by days 1 and 2 after IR injury)] and group 5 (TTIR + Mel + ADMSCs)], respectively. The dosages of Mel and ADMSCs utilized in the present study were based on our previous studies $[24,27,29,30]$.

\section{Histopathological finding of examination of testicular specimen after IR procedure}

Testes were dissected from the abdomen, immersed in $10 \%$ formalin for $24 \mathrm{~h}$, and embedded in paraffin. First, each testis was morphologically evaluated. Additionally, the tissue sections of $5 \mu \mathrm{m}$ were sliced and stained with haematoxylin and eosin (i.e., H\&E). The specimens of H\&E stain were carefully examined by light microscopy.

\section{Immunohistochemical (IHC) and immunofluorescent (IF) staining}

The procedures of IF and IHC staining were described in detail in our previous reports [24, 27, 29, 30]. For $\mathrm{IHC}$ and IF staining, rehydrated paraffin sections were first treated with $3 \% \mathrm{H}_{2} \mathrm{O}_{2}$ for 30 minutes and incubated with Immuno-Block reagent (BioSB) for 30 minutes at room temperature. Sections were then incubated with primary antibodies specifically against matrix metalloproteinase (MMP)-9 (1:200, Thermo Fisher), a-tubulin (1:500, Santa Cruz), myeloperoxidase (MPO) (1:200, Bio SB), androgen receptor (AR) (1:100, Santa Cruz), vimentin (1:500, Santa Cruz) and CD68 (1:200, Abcam), while sections incubated with the use of irrelevant antibodies served as controls. Three sections of the testicular specimen from each rat were analyzed. For quantification, three randomly selected HPFs (200x or 400x for IHC and IF studies) were analyzed in each section. The mean number of positively-stained cells per HPF for each animal was determined by summation of all numbers divided by 9 .

\section{Western blot analysis}

The procedure and protocol for Western blot analysis were based on our previous reports $[24,27,29,30]$. Briefly, equal amounts $(50 \mathrm{mg}$ ) of protein extracts were loaded and separated by SDS-PAGE using acrylamide gradients. After electrophoresis, the separated proteins were then transferred electrophoretically to a polyvinylidene difluoride (PVDF) membrane (Amersham Biosciences). Nonspecific sites were blocked by incubation of the membrane in blocking buffer [ $5 \%$ nonfat dry milk in T-TBS (TBS containing $0.05 \%$ Tween 20)] overnight. The membranes were then incubated with the indicated primary 
antibodies against cleaves caspase 3 (1:1000, Cell Signaling), cleaved poly(ADP-ribose) polymerase (cPARP) (1:1000, Cell Signaling), NOX-1 (1:750, Sigma), NOX-2 (1:1000, Sigma), phosphorylated (p)-Smad3 (1:1000, Cell Signaling), transforming growth factor (TGF)- -3 (1:1000, Abcam), mitochondrial Bax (1:1000, Abcam), cytosolic cytochrome $C(1: 1000, B D)$, mitochondrial cytochrome $C(1: 1000, B D)$, androgen receptor (AR) (1:1000, Abcam), and vimentin (1:1000, Abcam) for $1 \mathrm{~h}$ at room temperature. Horseradish peroxidase-conjugated anti-rabbit immunoglobulin IgG (1:2000, Cell Signaling) was used as a secondary antibody for one-hour incubation at room temperature. The washing procedure was repeated eight times within $1 \mathrm{~h}$. Immunoreactive bands were visualized by enhanced chemiluminescence (ECL; Amersham Biosciences) and exposed to Biomax L film (Kodak). For the purpose of quantification, ECL signals were digitized using Labwork software (UVP).

\section{Assessment of oxidative stress}

The procedure for assessing the protein expression of oxidative stress has been described in detail in our previous reports $[24,27,29,30]$. The Oxyblot Oxidized Protein Detection Kit was purchased from Chemicon (S7150). DNPH derivatization was carried out on $6 \mu \mathrm{g}$ of protein for 15 minutes according to the manufacturer's instructions. One-dimensional electrophoresis was carried out on $12 \%$ SDS/polyacrylamide gel after DNPH derivatization. Proteins were transferred to nitrocellulose membranes which were then incubated in the primary antibody solution (anti-DNP 1: 150) for 2h, followed by incubation in the secondary antibody solution (1:300) for 1 hour at room temperature. The washing procedure was repeated eight times within 40 minutes. Immunoreactive bands were visualized by enhanced chemiluminescence (ECL; Amersham Biosciences) which were then exposed to Biomax L film (Kodak). For quantification, ECL signals were digitized using Labwork software (UVP). For oxyblot protein analysis, a standard control was loaded on each gel.

\section{Histological measurement of testicular injuryand criteria for seminiferous tubules injury score}

The testicular specimens were sectioned at $5 \mu \mathrm{m}$ for light microscopy of H\&E stain for each animal. Three testicular sections from each rat were analyzed and three randomly selected high-power fields (HPFs; 200x) were examined in each section. The morphologic destruction score of seminiferous tubules was evaluated in a blinded fashion. The following scoring system was adopted: $0=$ no detectable morphologic destruction; $1=<15 \%$ of morphologic destruction; $2=15-25 \%$ of morphologic destruction; 3 $=26-50 \%$ of morphologic destruction; $4=51-75 \%$ of morphologic destruction; $5=76-100 \%$ of morphologic destruction /HPF.

\section{Statistical analysis}

Quantitative data were expressed as mean \pm SD. Statistical analysis was adequately performed by ANOVA followed by Bonferroni multiple-comparison post hoc test. SAS statistical software for Windows version 8.2 (SAS institute, Cary, NC, USA) was utilized for the analysis. A probability value $<0.05$ was considered as statistical significance. 


\section{Results}

The protein expressions of apoptotic and oxidative-stress biomarkers by $72 \mathrm{~h}$ after IR procedure (Fig. 1)

To determine whether the apoptosis and oxidative stress signalings were upregulated after IR procedure, the Western blot analysis was performed. The result showed that the protein expressions of mitochondrial Bax, cleaved caspase 3 and cleaved PARP, three indices of apoptosis, were lowest in group 1 (i.e., SC), highest in group 2 (TTIR only) and significantly lower in group 5 (TTIR + Mel + ADMSCs) than in groups $3(T T I R+M e l)$ and $4(T T I R+A D M S C s)$, but they exhibited no difference between groups 3 and 4. Consistently, the protein expressions of NOX-1, NOX-2 and oxidized protein, three indicators of oxidative stress, displayed an identical pattern of apoptosis.

\section{Protein expressions of fibrotic and mitochondrial-damaged markers by $72 \mathrm{~h}$ after IR procedure (Fig. 2)}

To elucidate the therapeutic impact of Mel-ADMCSs on regulating the fibrosis and DNA-damaged signalings, the Western blot was utilized. The result showed that the protein expressions of Smad3 and TGF- $\beta$, two indicators of fibrosis, were lowest in group 1, highest in group 2 and significantly lower in group 5 than in groups 3 and 4, but they showed no difference between the latter two groups. Additionally, the protein expression of cytosolic cytochrome $\mathrm{C}$, a mitochondria damage biomarker, exhibited a similar pattern whereas, the protein expression of mitochondrial cytochrome $C$, an indicator of mitochondrial integrity, exhibited an opposite pattern of fibrosis among the five groups.

\section{Protein and cellular expressions of androgen receptor and vimentin by $72 \mathrm{~h}$ after IR procedure (Fig. 3)}

We further evaluated the expressions of vimentin and AR, two indicators of integrity of testis and its apparatus, with Western blot and microscope. The result showed that the protein and cellular expressions of vimentin and AR were highest in group 1, lowest in group 2 and significantly higher in group 5 than in groups 3 and 4, but they were similar in groups 3 and 4 .

\section{Scoring for identification of loss of seminiferous tubule epithelium (STE) by $72 \mathrm{~h}$ after IR procedure (Fig. 4)}

To assess the therapeutic impact of Mel-ADMCSs on protecting the seminiferous tubules (ST), the microscopic examination was performed. The result of H.E. stain demonstrated that the thickness of ST, an indicator of intactness of the epithelium, was highest in group 1, lowest in group 2 and significantly higher in group 5 than in groups 3 and 4 , but it was similar between groups 3 and 4 .

On the other hand, the morphologic destruction score of ST, an indicator of ST damage, exhibited an opposite pattern to the thickness of ST among the groups.

\section{The cellular expression of Sertoli cells in testis by $72 \mathrm{~h}$ after IR procedure (Fig. 5)}

Next, we investigated the protective effect of Mel-ADMCSs on the integrity of the Sertoli cells, a part of a seminiferous tubule participating in the process of spermatogenesis and the production of sperm. The 
microscopic finding of H.E. stain demonstrated that the number of Sertoli cells was highest in group 1 , lowest in group 2 and significantly higher in group 5 than in groups 3 and 4, but it was similar between groups 3 and 4.

\section{The cellular expression of alpha-microtubule and CD68 in testis by $72 \mathrm{~h}$ after IR procedure (Fig. 6)}

It is well known that alpha-microtubule (a-MT)-based cytoskeleton is crucial to confer spermatid and organelle transport. The result of the present study demonstrated that the number of a-MT was highest in group 1, lowest in group 2 and significantly higher in group 5 than in groups 3 and 4, but it was similar between groups 3 and 4, suggesting that combined Mel-ADMCSs was superior to either one alone for protecting this testicular apparatus against IR damage. On the other hand, the cellular expression of $\mathrm{CD68}$, an indicator of cellular level of inflammation, displayed an opposite pattern of a-MT among the groups, implicating that Mel-ADMCSs was superior to either one alone for protecting the testis against inflammatory damage.

\section{The cellular level of inflammatory reaction in testis by $72 \mathrm{~h}$ after IR procedure (Fig. 7)}

Undoubtedly, inflammatory reaction plays a key role on tissue/organ damage after ischemic or IR attack. In the present study, IHC stain was utilized for delineating the impact of Mel-ADMCSs on restraining the IR from damaging the testis. As we expected, the cellular expression of MMP-9 and MPO, two indicators of inflammation, were lowest in group 1, highest in group 2 and significantly lower in group 5 than in groups 3 and 4 , but they did not differ between groups 3 and 4 .

\section{Discussion}

This study which investigated the therapeutic impact of Mel-ADMCSs on protecting the testis against TTIR injury yielded several striking preclinical implications. First, we successfully created TTIR animal model for answering key questions in the current study. Second, the result of the present study demonstrated that Mel was comparable to ADMSCs for attenuating the IR-induced testicular injury. Third, the result of the present study further showed that combined Mel and ADMCSs was superior to either one alone on protecting the testis against the TTIR injury, highlighting that this therapeutic management may be potential for the TTIR injured patients, especially for those who are refractory to the conventional therapy for preserving fertility.

Abundant clinical studies have identified that testicular torsion is a serious urological emergency that is most frequently encountered in the newborns, children and adolescent males [1, 2]. Despite utmost importance in rapid diagnosis and prompt treatment [3-5], majority of the patients still have profound damage due to the fact that testicular is an intrinsically vulnerable organ, resulting in the potentially grave sequelae of infertility and subfertility. This suggests that the conventional interventions are indeed lacking much to be desired, raising the need of considering a new and safe supplementary treatment to ameliorate the development of grave sequelae of infertility and subfertility in acute TTIR patients just after the surgical procedure. 
An association between organ IR injury and generation of oxidative stress and vigorous inflammatory reaction has been extensively investigated [24, 29-32]. Studies have further demonstrated that MelADMSCs therapy effectively protected the IR tissue/organs mainly through ameliorating the molecularcellular perturbations [29-31]. An essential finding in the present study was that the inflammatory and oxidative stress biomarkers were markedly increased in TTIR animals than in those of SC group. In this way, our findings, in addition to being consistent with the findings of the previous studies [24, 29-32], could explain the testicular macro- and micro-structural damage in this TTIR animals. However, our results further demonstrated that Mel and ADMSCs were comparable and combined Mel-ADMSCs therapy was superior to either one on suppressing these molecular-cellular perturbations and protecting the integrity of testicular architectures. Accordingly, our findings corroborated the findings of these previous reports [29-31].

A link between the higher the protein expressions of apoptotic, fibrotic and mitochondrial-damaged biomarkers in IR organs, the more severe the damage of the organs [24, 26, 29-31], implicating that these are useful prognostic biomarkers in setting of IR injury. A principal finding in the present study was that the protein expressions of apoptosis, fibrosis and cytosolic cytochrome $\mathrm{C}$ (i.e., a mitochondrial damaged marker) were substantially increased in TTIR group than in the SC group. Our findings, in addition to being consistent with the findings of those previous studies [24, 26, 29-31], could at least in part explained why the testicular macro- and micro-structural were deeply destructed in TTIR animals. Importantly, these parameters were notably improved by either Mel or AMDSCs treatment, and further remarkably reversed by combined Mel-ADMSCs treatment.

The most important finding in the present study was that numbers of Sertoli cells, AR cells, seminiferous tubule epithelium (i.e., the architectures of testis) and the vimentin (i.e., ultrastructure of the cells) as well as the thickness of epithelium were substantially reduced in TTIR group than in SC group. However, these testicular damaged parameters were notably alleviated by Mel or ADMSCs therapy and further significantly reversed by combined Mel-ADMSCs therapy, highlighting that the Mel-ADMSCs treatment should be earnestly considered as a potential option for those patients who suffer from disastrously TTIR injury.

\section{Study Limitation}

This study has limitations. First, although the results of the present study were attractive and promising, the study period was relatively short (i.e., the study period was only $72 \mathrm{~h}$ ). Thus, the long-term impact of Mel-ADMSCs therapy on protecting the testis against TTIR damage remains uncertain. Second, despite the molecular-cellular level of AR was significantly preserved in TTIR animals undergoing Mel-ADMSCs, the functional integrity of these TTIR animals was not assessed. Accordingly, the fertility ability (defined as the final success of therapy by Mel-ADMSCs) of these animals is still currently unclear.

In conclusion, the result of the present study demonstrated that combined Mel-ADMSCs therapy offered additional benefit on protecting the testis against TTIR injury. 


\section{Abbreviations}

a-MT: alpha-microtubule; ADMSCs: adipose-derived mesenchymal stem cells; AR: androgen receptor; DNA: deoxyribonucleic acid; ECL: enhanced chemiluminescence; H\&E stain: haematoxylin and eosin stain; HPFs: High-power fields; IF: immunofluorescent; IHC: immunohistochemistry; IR: ischemiareperfusion injury; Mel: melatonin; MMP: matrix metalloproteinase; MPO: myeloperoxidase; NOX: NADPH oxidase; PARP: poly (ADP-ribose) polymerase; PVDF: polyvinylidene difluoride; ROS: reactive oxygen species; SC: sham-operated control; SDS-PAGE: sodium dodecyl sulfate-Polyacrylamide gel electrophoresis; ST: seminiferous tubules; TBS: Tris-buffered saline; TGF-ß: transforming growth factor Beta; TTIR: testicular torsion-induced ischemia-reperfusion injury.

\section{Declarations}

\section{Acknowledgments}

This study was supported by a program grant from Chang Gung Memorial Hospital, Chang Gung University (Grant number: CMRPG8H0781).

\section{Author Contributions}

Investigation, Yen-Ta Chen, Fei-Chi Chuang, Chih-Chao Yang, Pei-Hsun Sung, Yi-Ching Chu, Chi-Ruei Huang; Methodology, Yen-Ta Chen, Fei-Chi Chuang, Chih-Chao Yang, Pei-Hsun Sung and Kuan-Hui Huang; Supervision, Kuan-Hui Huang and Hon-Kan Yip; Writing - review \& editing, John Y. Chiang, KuanHui Huang and Hon-Kan Yip.

\section{Funding}

This study was supported by a program grant from Chang Gung Memorial Hospital, Chang Gung University (Grant number: CMRPG8H0781).

\section{Availability of data and materials}

The data that support the findings of this study are available from the corresponding authors upon reasonable request.

\section{Ethics approval and consent to participate}

All animal experimental procedures were approved by the Institutional Animal Care and Use Committee at Kaohsiung Chang Gung Memorial Hospital (Affidavit of Approval of Animal Use Protocol No. 2017110201) and performed in accordance with the Guide for the Care and Use of Laboratory Animals, $8^{\text {th }}$ edition (NIH publication No. 85-23, National Academy Press, Washington, DC, USA, revised 2011).

\section{Consent for publication}


Not applicable.

\section{Conflicts of interest}

All authors have read the journal's policy on disclosure of potential conflicts of interest and the journal's authorship agreement. The authors declare that they have no conflicts of interest. The article has been reviewed by and approved by all named authors.

\section{References}

[1] Karaguzel E, Kadihasanoglu M and Kutlu O. Mechanisms of testicular torsion and potential protective agents. Nat Rev Urol 2014; 11: 391-399.

[2] Howe AS, Vasudevan V, Kongnyuy $M$, et al. Degree of twisting and duration of symptoms are prognostic factors of testis salvage during episodes of testicular torsion. Transl Androl Urol 2017; 6: $1159-1166$.

[3] Turner TT and Brown KJ. Spermatic cord torsion: loss of spermatogenesis despite return of blood flow. Biol Reprod 1993; 49: 401-407.

[4] Unsal A, Devrim E, Guven C, et al. Propofol attenuates reperfusion injury after testicular torsion and detorsion. World J Urol 2004; 22: 461-465.

[5] Cay A, Alver A, Kucuk M, et al. The effects of N-acetylcysteine on antioxidant enzyme activities in experimental testicular torsion. J Surg Res 2006; 131: 199-203.

[6] Prillaman HM and Turner TT. Rescue of testicular function after acute experimental torsion. J Urol 1997; 157: 340-345.

[7] Alp BF, Cebi G, Ozdemir A, et al. Hyperbaric oxygen treatment for unilateral testicular torsion in a child. Diving Hyperb Med 2014; 44: 161-162.

[8] Dokmeci D, Inan M, Basaran UN, et al. Protective effect of L-carnitine on testicular ischaemiareperfusion injury in rats. Cell Biochem Funct 2007; 25: 611-618.

[9] Koksal M, Oguz E, Baba F, et al. Effects of melatonin on testis histology, oxidative stress and spermatogenesis after experimental testis ischemia-reperfusion in rats. Eur Rev Med Pharmacol Sci 2012; 16: $582-588$.

[10] Shokoohi M, Shoorei $\mathrm{H}$, Soltani M, et al. Protective effects of the hydroalcoholic extract of Fumaria parviflora on testicular injury induced by torsion/detorsion in adult rats. Andrologia 2018; 50: e13047.

[11] Visser AJ and Heyns CF. Testicular function after torsion of the spermatic cord. BJU Int 2003; 92: 200-203. 
[12] Filho DW, Torres MA, Bordin AL, et al. Spermatic cord torsion, reactive oxygen and nitrogen species and ischemia-reperfusion injury. Mol Aspects Med 2004; 25: 199-210.

[13] Dokmeci D. Testicular torsion, oxidative stress and the role of antioxidant therapy. Folia Med (Plovdiv) 2006; 48: 16-21.

[14] Jeong SJ, Choi WS, Chung JS, et al. Preventive effects of cyclosporine a combined with prednisolone and melatonin on contralateral testicular damage after ipsilateral torsion-detorsion in pubertal and adult rats. J Urol 2010; 184: 790-796.

[15] Yildiz H, Durmus AS, Simsek $H$, et al. Protective effect of sildenafil citrate on contralateral testis injury after unilateral testicular torsion/detorsion. Clinics (Sao Paulo) 2011; 66: 137-142.

[16] Parlaktas BS, Atilgan D, Ozyurt H, et al. The biochemical effects of ischemia-reperfusion injury in the ipsilateral and contralateral testes of rats and the protective role of melatonin. Asian $\mathrm{J}$ Androl 2014; 16 : 314-318.

[17] Fernandez A, Ordonez R, Reiter RJ, et al. Melatonin and endoplasmic reticulum stress: relation to autophagy and apoptosis. J Pineal Res 2015; 59: 292-307.

[18] Galano A, Tan DX and Reiter RJ. Melatonin as a natural ally against oxidative stress: a physicochemical examination. J Pineal Res 2011; 51: 1-16.

[19] Galano A, Tan DX and Reiter RJ. On the free radical scavenging activities of melatonin's metabolites, AFMK and AMK. J Pineal Res 2013; 54: 245-257.

[20] Zhang HM and Zhang Y. Melatonin: a well-documented antioxidant with conditional pro-oxidant actions. J Pineal Res 2014; 57: 131-146.

[21] Kang JW, Koh EJ and Lee SM. Melatonin protects liver against ischemia and reperfusion injury through inhibition of toll-like receptor signaling pathway. J Pineal Res 2011; 50: 403-411.

[22] Sun CK, Lee FY, Kao YH, et al. Systemic combined melatonin-mitochondria treatment improves acute respiratory distress syndrome in the rat. J Pineal Res 2015; 58: 137-150.

[23] Garcia JJ, Lopez-Pingarron L, Almeida-Souza P, et al. Protective effects of melatonin in reducing oxidative stress and in preserving the fluidity of biological membranes: a review. J Pineal Res 2014; 56 : 225-237.

[24] Chen HH, Lin KC, Wallace CG, et al. Additional benefit of combined therapy with melatonin and apoptotic adipose-derived mesenchymal stem cell against sepsis-induced kidney injury. J Pineal Res 2014; 57: 16-32. 
[25] Tang XL, Rokosh G, Sanganalmath SK, et al. Intracoronary administration of cardiac progenitor cells alleviates left ventricular dysfunction in rats with a 30-day-old infarction. Circulation 2010; 121: 293-305.

[26] Leu S, Sun CK, Sheu JJ, et al. Autologous bone marrow cell implantation attenuates left ventricular remodeling and improves heart function in porcine myocardial infarction: an echocardiographic, sixmonth angiographic, and molecular-cellular study. Int J Cardiol 2011; 150: 156-168.

[27] Chen YT, Chiang HJ, Chen $\mathrm{CH}$, et al. Melatonin treatment further improves adipose-derived mesenchymal stem cell therapy for acute interstitial cystitis in rat. J Pineal Res 2014; 57: 248-261.

[28] Minnerup J, Wersching H, Teuber A, et al. Outcome After Thrombectomy and Intravenous Thrombolysis in Patients With Acute Ischemic Stroke: A Prospective Observational Study. Stroke 2016; 47: 1584-1592.

[29] Yip HK, Chang YC, Wallace CG, et al. Melatonin treatment improves adipose-derived mesenchymal stem cell therapy for acute lung ischemia-reperfusion injury. J Pineal Res 2013; 54: 207-221.

[30] Chen HH, Chang CL, Lin KC, et al. Melatonin augments apoptotic adipose-derived mesenchymal stem cell treatment against sepsis-induced acute lung injury. Am J Transl Res 2014; 6: 439-458.

[31] Lin KC, Yip HK, Shao PL, et al. Combination of adipose-derived mesenchymal stem cells (ADMSC) and ADMSC-derived exosomes for protecting kidney from acute ischemia-reperfusion injury. Int $\mathrm{J}$ Cardiol 2016; 216: 173-185.

[32] Wei SM, Yan ZZ and Zhou J. Beneficial effect of taurine on testicular ischemia-reperfusion injury in rats. Urology 2007; 70: 1237-1242.

\section{Figures}


(A)
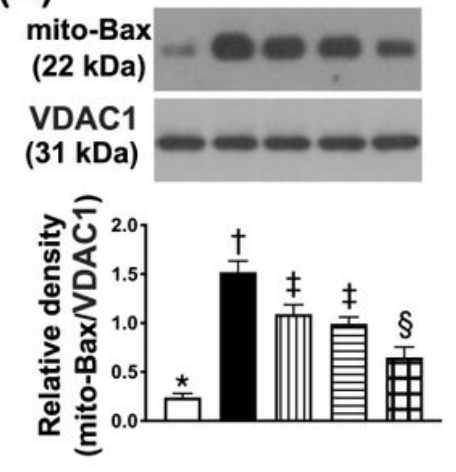

(E)

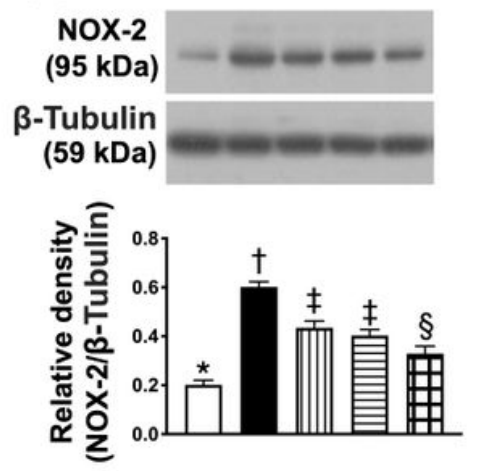

(B)
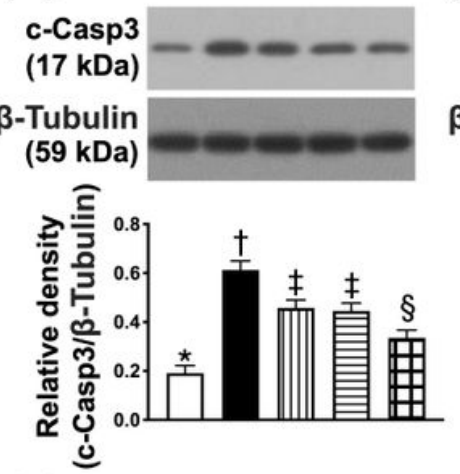

(F) M.W. $\square$ 口四目团 DNP st.

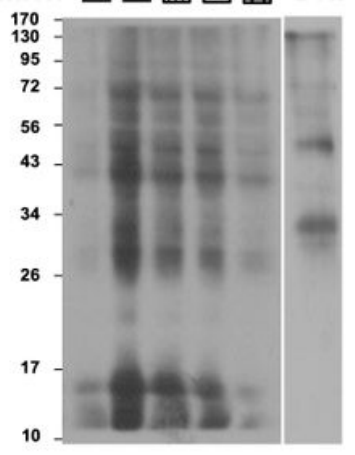

(C)

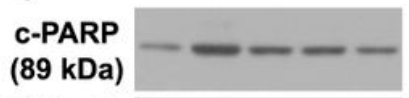

$\beta$-Tubulin

$(59 \mathrm{kDa})$
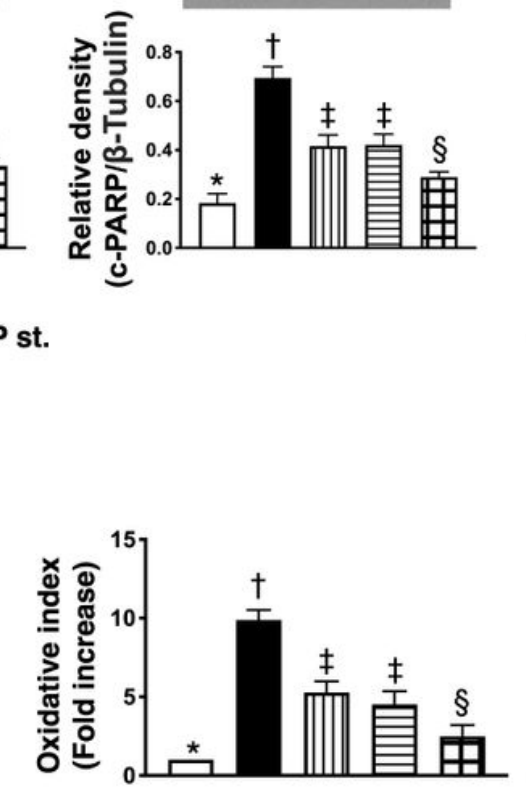

(D)
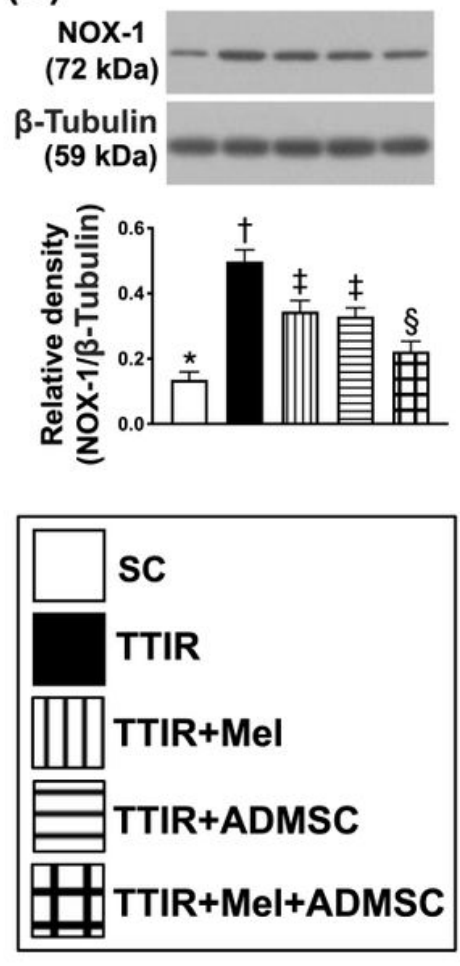

Figure 1

Mel-ADMSCs therapy suppressed the protein expressions of apoptosis and oxidative stress by $72 \mathrm{~h}$ after TTIR procedure A) Protein expressions of mitochondrial (mito)-Bax, * vs. other groups with different symbols $(\dagger, \ddagger, \S), p<0.0001$. B) Protein expression of cleaved caspase 3 (c-Casp3), * vs. other groups with different symbols $(\dagger, \ddagger, \S), p<0.0001$. C) Protein expression of and cleaved poly (ADP-ribose) polymerase (c-PARP), * vs. other groups with different symbols $(\dagger, \ddagger, \S), p<0.0001$. D) Protein expression of NOX-1, * vs. other groups with different symbols $(t, \neq, \S), p<0.0001$. E) Protein expression of NOX-2, * vs. other groups with different symbols $(t, \neq, \S), p<0.0001$. F) Oxidized protein expression, * vs. other groups with different symbols $(\dagger, \ddagger, \S), p<0.0001$. (Note: left and right lanes shown on the upper panel represent protein molecular weight marker and control oxidized molecular protein standard, respectively). M.W. = molecular weight; DNP = 1-3 dinitrophenylhydrazone. All statistical analyses were performed by one-way ANOVA, followed by Bonferroni multiple comparison post hoc test ( $n=6$ for each group). Symbols $(*, t, \neq$, $\S$ ) indicate significance (at 0.05 level). SC = sham-operated control; TTIR = testicular torsion-induced ischemia-reperfusion; Mel = melatonin; $\mathrm{ADMSCs}$ = adipose-derived mesenchymal stem cells. 
(A)

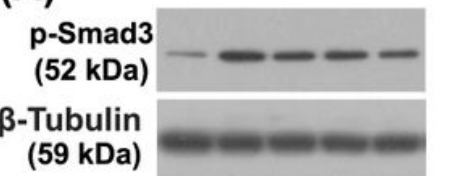

(59 kDa)

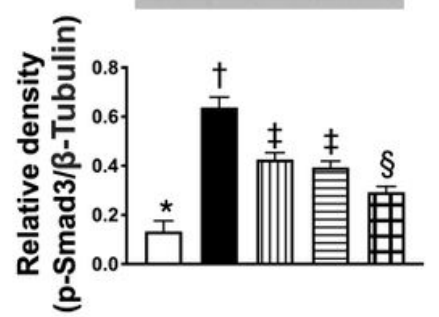

(B)

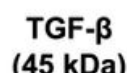

$\beta$-Tubulin

$(59 \mathrm{kDa})$

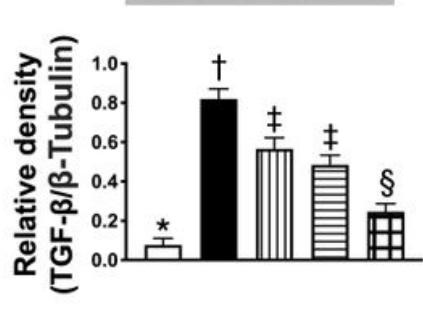

(C)

cyt-Cyto-C

(15 kDa)

$\beta$-Tubulin

(59 kDa)

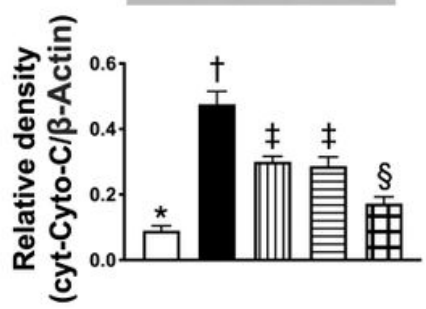

(D)

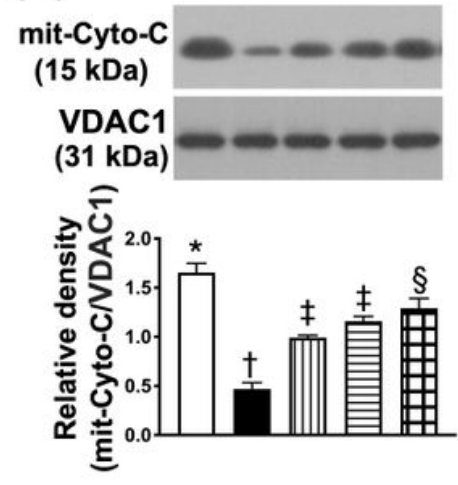

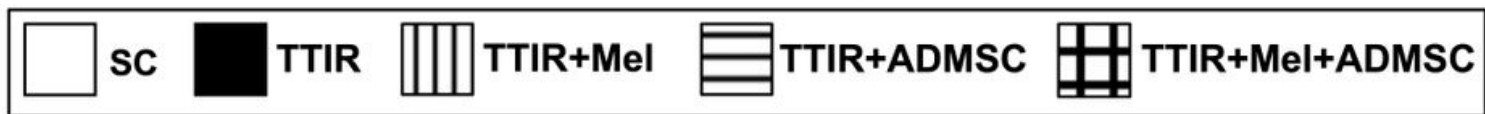

Figure 2

Mel-ADMSCs therapy inhibited the protein expressions of fibrosis and mitochondrial-damaged biomarker by $72 \mathrm{~h}$ after TTIR procedure A) Protein expression of Smad3, * vs. other groups with different symbols $(t, \neq, \S), p<0.0001$. B) Protein expression of transforming growth factor (TGF)- $\beta$, * vs. other groups with different symbols $(\dagger, \ddagger, \S), p<0.0001$. C) Protein expression of cytosolic cytochrome $C$ (cyt-Cyto-C), * vs. other groups with different symbols $(t, \ddagger, \S), p<0.0001$. D) Protein expression of mitochondrial cytochrome $C$ (mit-Cyto-C), * vs. other groups with different symbols $(t, \ddagger, \S), p<0.0001$. All statistical analyses were performed by one-way ANOVA, followed by Bonferroni multiple comparison post hoc test ( $n=6$ for each group). Symbols $(*, \dagger, \neq, \S)$ indicate significance (at 0.05 level). SC = sham-operated control; TTIR = testicular torsion-induced ischemia-reperfusion; $\mathrm{Mel}=$ melatonin; ADMSCs = adipose-derived mesenchymal stem cells. 
(A)
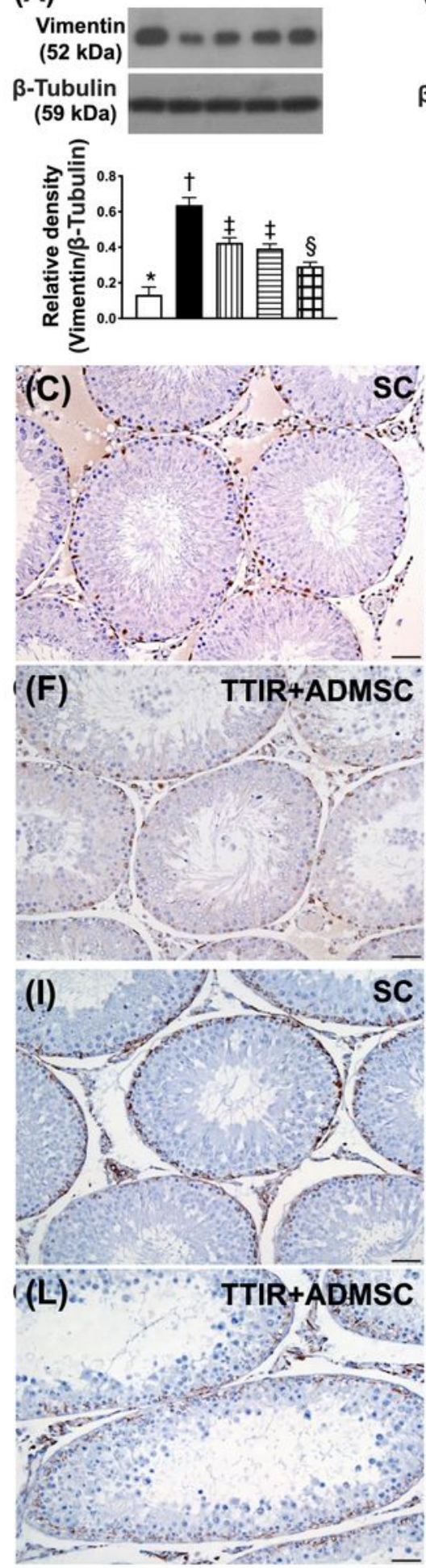

(B)
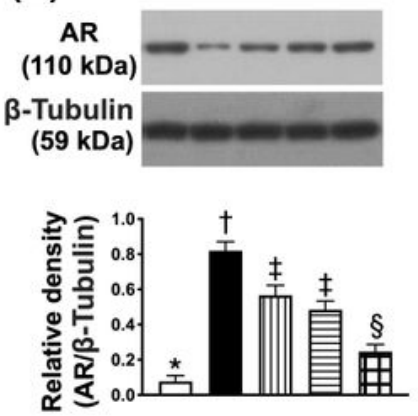

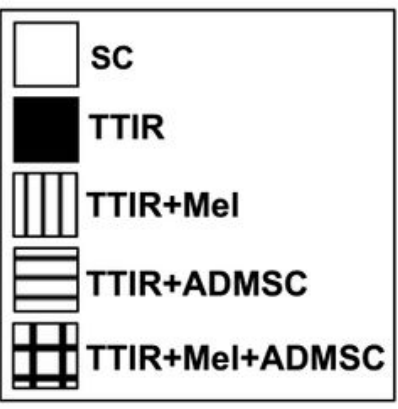

(D)

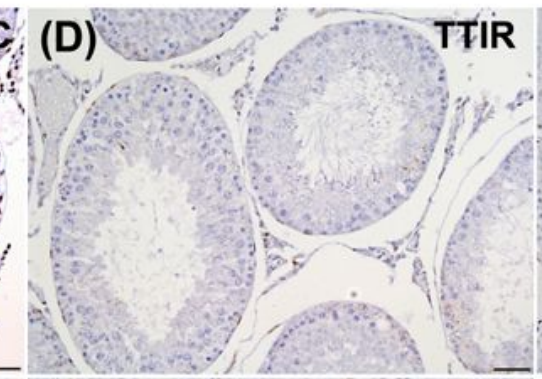

(E)

TTIR+Mel
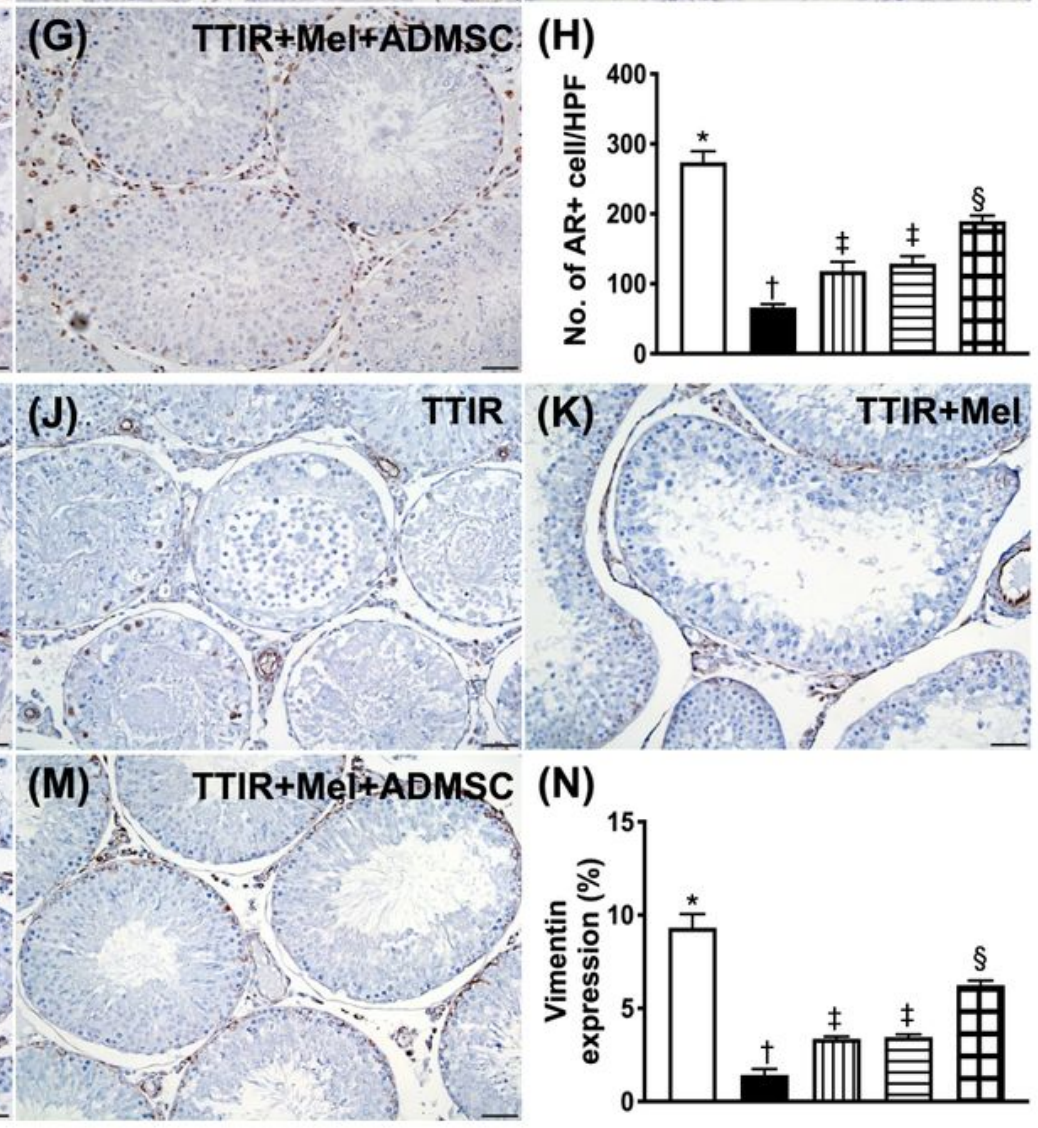

(N)

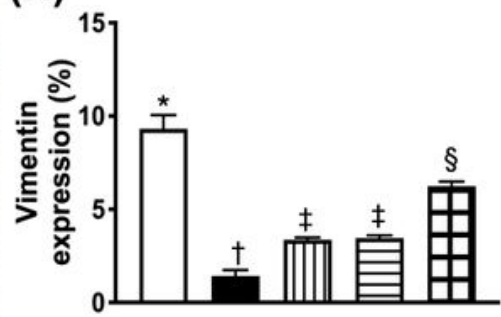

\section{Figure 3}

Mel-ADMSCs therapy upregulated the protein and cellular expressions of androgen receptor and vimentin by $72 \mathrm{~h}$ after TTIR procedure A) Protein expression of vimentin, * vs. other groups with different symbols $(\dagger, \ddagger, \S), p<0.0001$. B) Protein expression of androgen receptor (AR), * vs. other groups with different symbols $(\dagger, \neq, \S), p<0.0001$. C to $G)$ Illustrating the immunohistochemical (IHC) stain for identification of AR+ cells (gray color). H) Analytical result of number of AR-positively stained cells, * vs. other groups with 
different symbols $(\dagger, \ddagger, \S), p<0.0001$. I to M) Showing the IHC stain for identification of vimentin + cells (gray color). N) Analytical result of number of vimentin-positively stained cells, * vs. other groups with different symbols $(\dagger, \ddagger, \S), p<0.0001$. All statistical analyses were performed by one-way ANOVA, followed by Bonferroni multiple comparison post hoc test ( $n=6$ for each group). Symbols $\left({ }^{*}, t, \neq, \S\right)$ indicate significance (at 0.05 level). SC = sham-operated control; TTIR = testicular torsion-induced ischemiareperfusion; Mel = melatonin; $\mathrm{ADMSC}$ = adipose-derived mesenchymal stem cells.

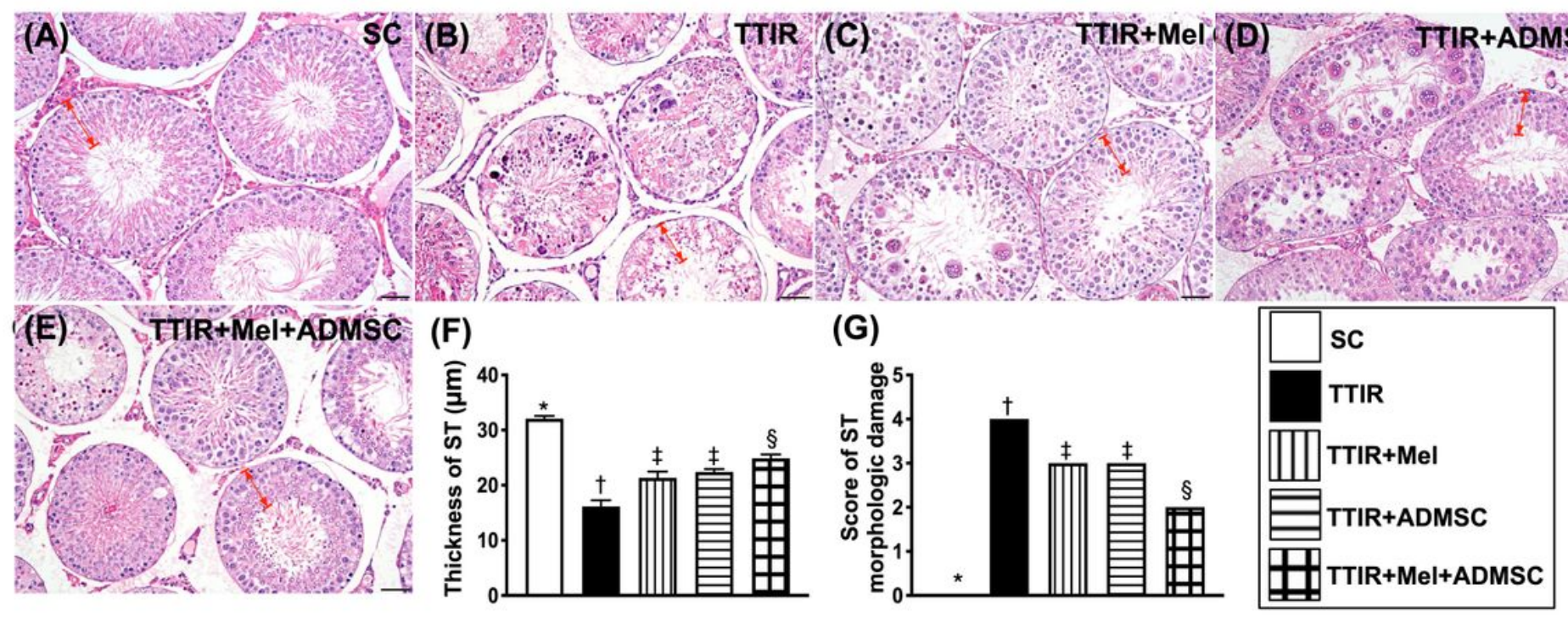

\section{Figure 4}

Using histopathological analysis and scoring for identification of seminiferous tubule damage by $72 \mathrm{~h}$ after IR procedure A to E) Illustrating the microscopic (200x) of H.E. stain for identification of histological feature (i.e., cross-section) of testis. The architectural feature of seminiferous tubules (ST) (red color) was remarkably destructed in TTIR groups as compared with the other groups. F) The analytical result of the thickness of ST (red color of double arrows), * vs. other groups with different symbols $(t, \neq, \S), p<0.0001$. G) Score of the severity of ST morphologic damage, * vs. other groups with different symbols $(t, \ddagger, \S)$, $p<0.0001$. HPF $=$ high-power field. All statistical analyses were performed by one-way ANOVA, followed by Bonferroni multiple comparison post hoc test ( $n=6$ for each group). Symbols $(*,+, \neq, \S)$ indicate significance (at 0.05 level). SC = sham-operated control; TTIR = testicular torsion-induced ischemiareperfusion; $\mathrm{Mel}=$ melatonin; $\mathrm{ADMSC}$ = adipose-derived mesenchymal stem cells. 


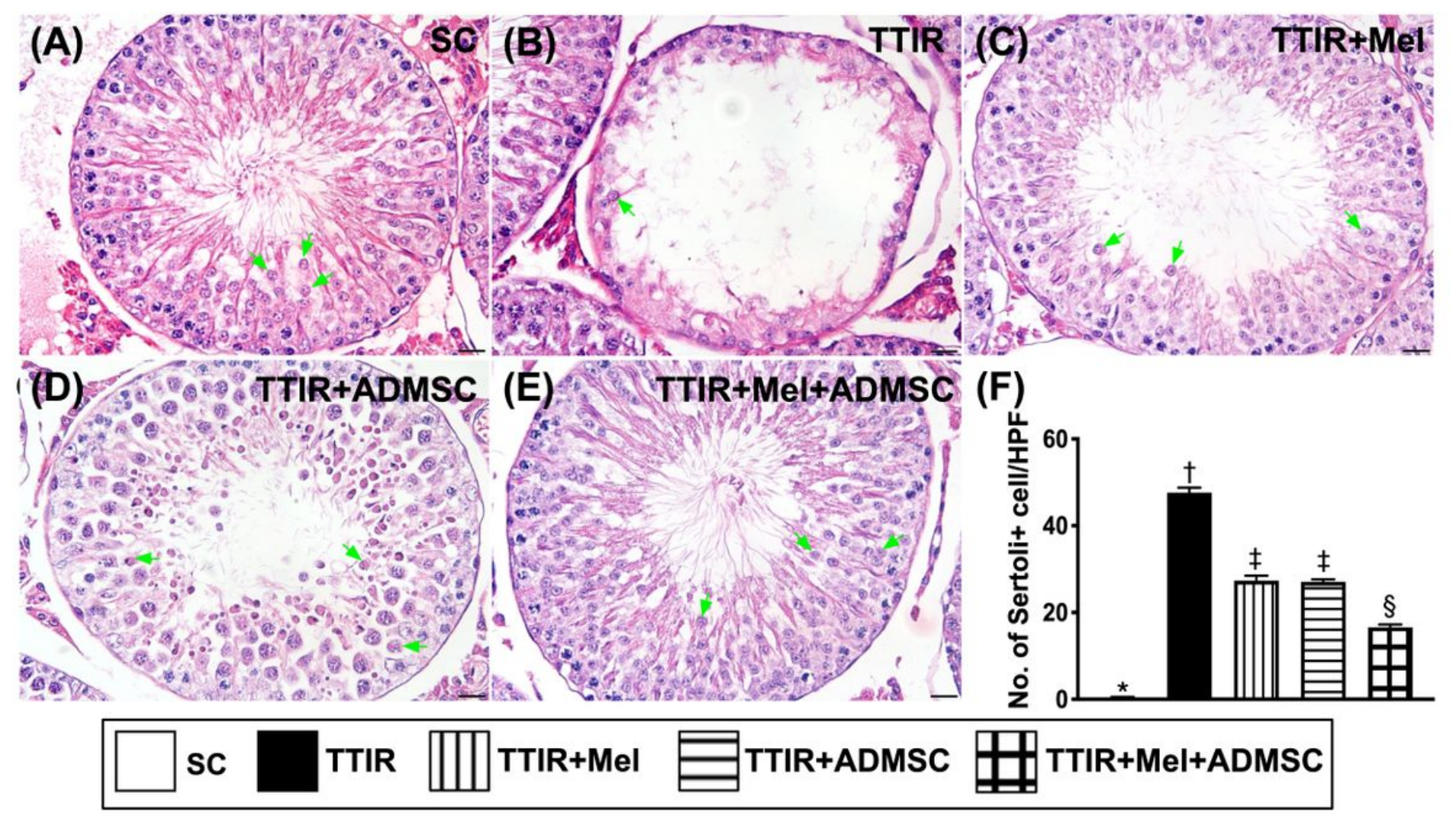

Figure 5

Mel-ADMSCs therapy effectively protected Sertoli cells against TTIR injury by $72 \mathrm{~h}$ after IR procedure A to E) Illustrating the microscopic (400x) finding of H.E. stain for identification of histological feature (i.e., cross-section) of Sertoli cells (green arrows). The result showed that number of Sertoli cells was notably reduced in TTIR groups than in other groups. F) The analytical result of the number of Sertoli cells, * vs. other groups with different symbols $(t, \neq, \S), p<0.0001$. HPF $=$ high-power field. All statistical analyses were performed by one-way ANOVA, followed by Bonferroni multiple comparison post hoc test $(\mathrm{n}=6$ for each group). Symbols $(*,+, \neq, \S)$ indicate significance (at 0.05 level). SC = sham-operated control; TTIR = testicular torsion-induced ischemia-reperfusion; $\mathrm{Mel}=$ melatonin; $\mathrm{ADMSC}$ = adipose-derived mesenchymal stem cells. 


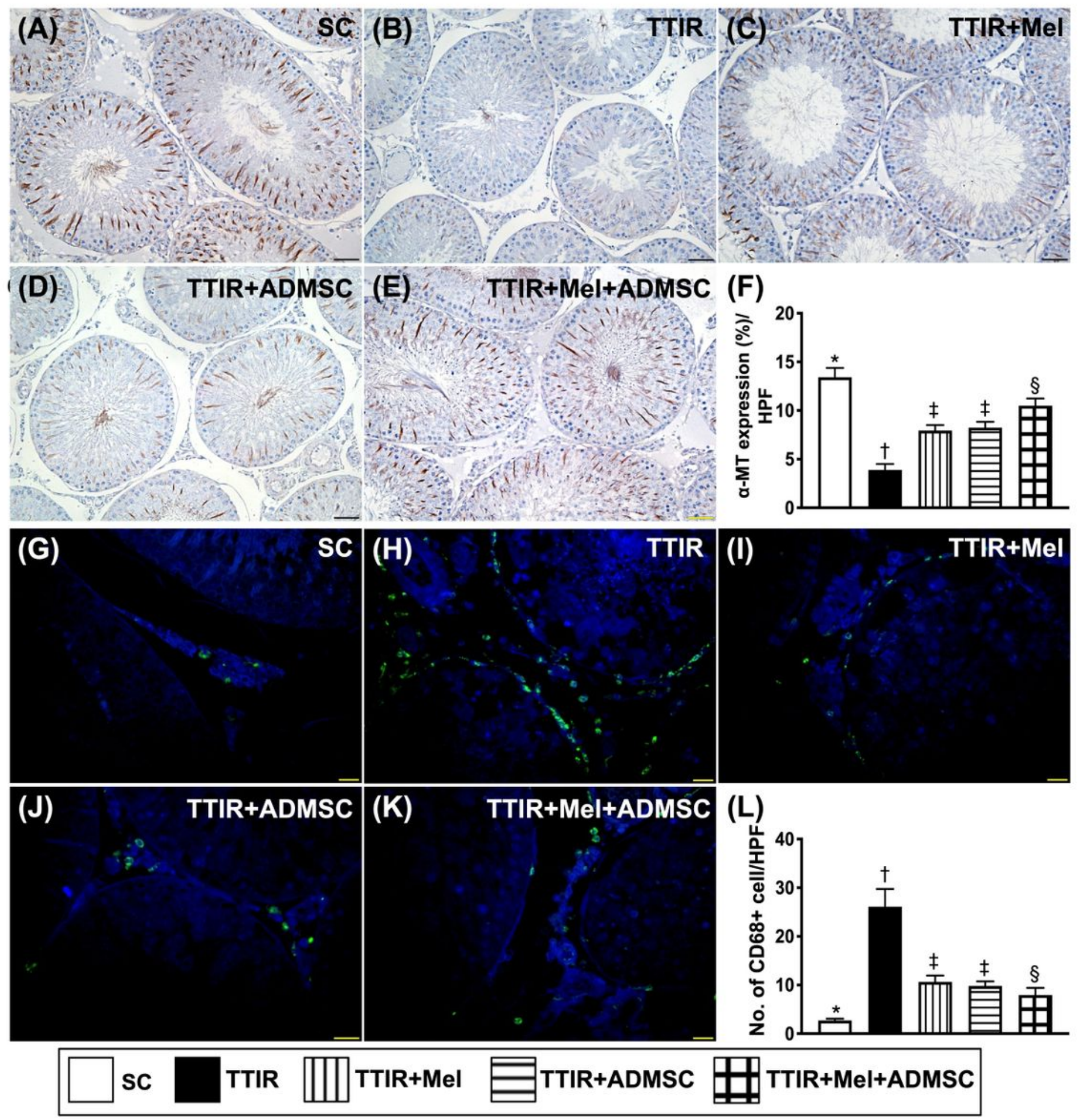

Figure 6

Mel-ADMSCs therapy preserved the integrity of alpha-microtubule and suppression of cellular expression of CD68 in testis by $72 \mathrm{~h}$ after IR procedure A to E) Illustrating the microscopic finding (400x) of immunohistochemical stain for identification of the expression of alpha-microtubule (a-MT) (spindle shape, gray color). F) Analytical result of number of a-MT, * vs. other groups with different symbols ( $\dagger, \neq$, $\S), p<0.0001$. G to K) Illustrating the immunofluorescent (IF) microscopic finding (400x) for identification 
of CD68-positively stained cells (green color). L) Analytical result of number of CD68+ cells, * vs. other groups with different symbols $(\dagger, \ddagger, \S), p<0.0001 . \mathrm{HPF}=$ high-power field. All statistical analyses were performed by one-way ANOVA, followed by Bonferroni multiple comparison post hoc test $(n=6$ for each group). Symbols $(*,+, \neq, \S)$ indicate significance (at 0.05 level). SC = sham-operated control; TTIR = testicular torsion-induced ischemia-reperfusion; $\mathrm{Mel}=$ melatonin; $\mathrm{ADMSCs}=$ adipose-derived mesenchymal stem cells.
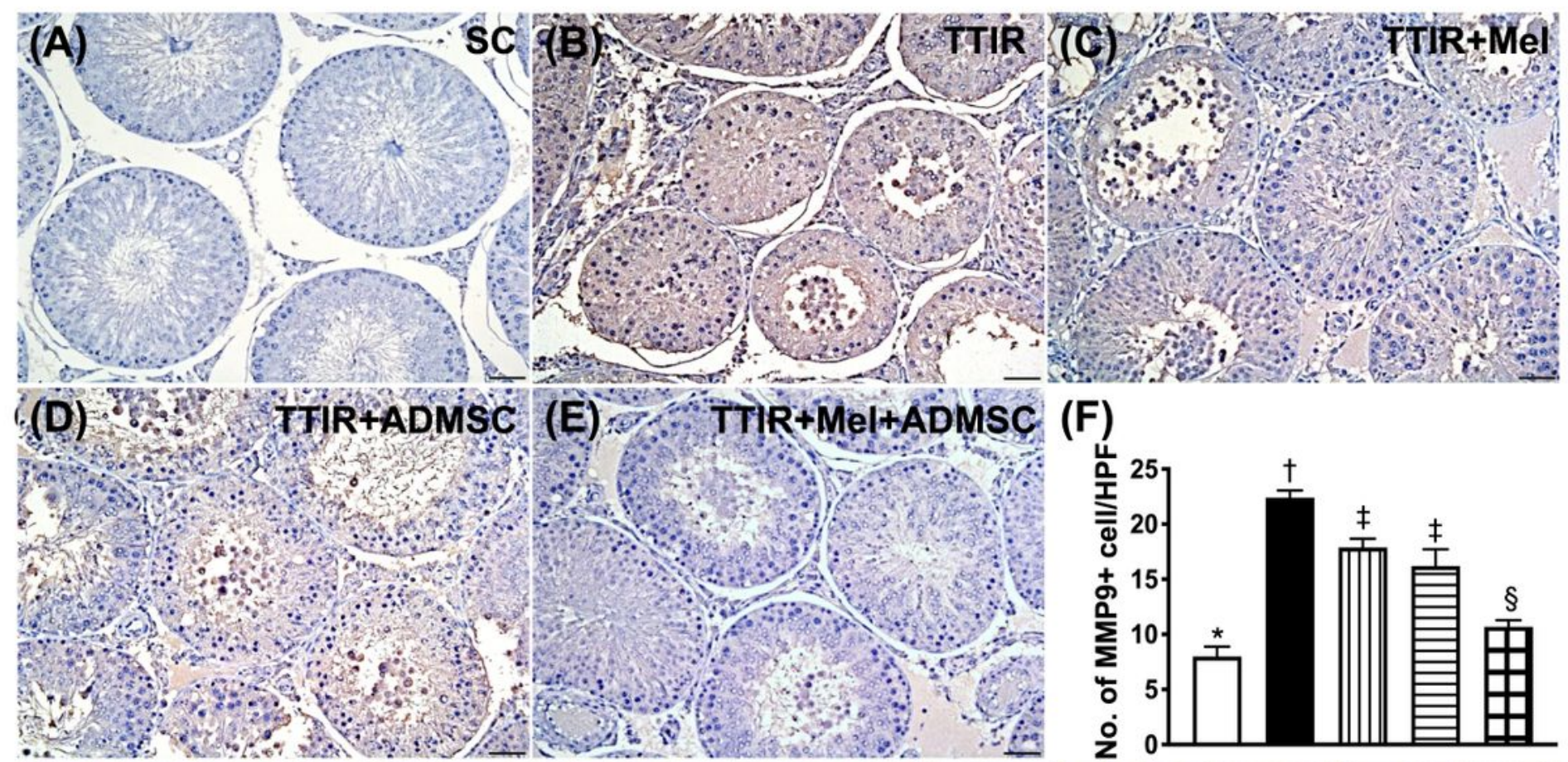

(F)
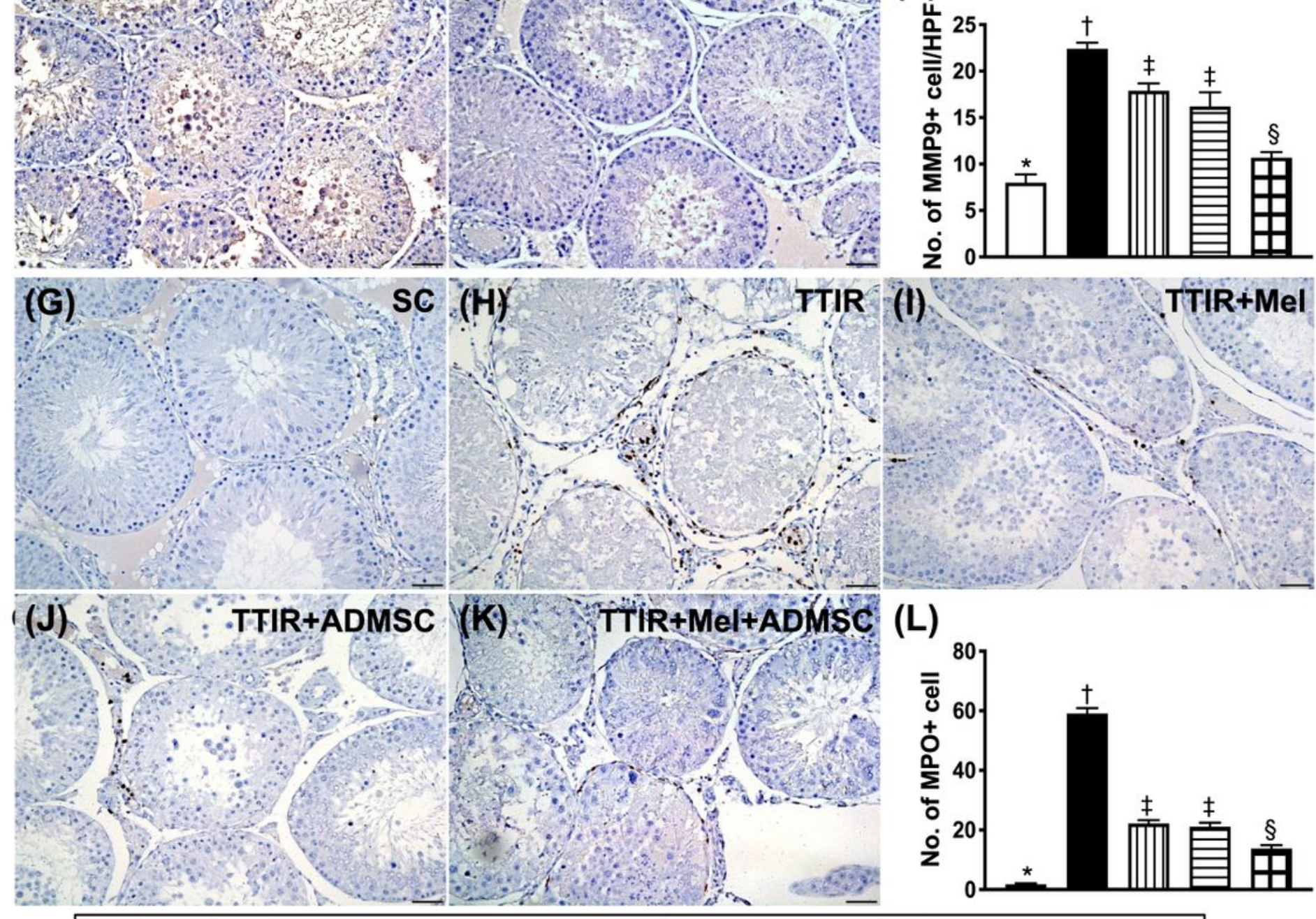

(L)

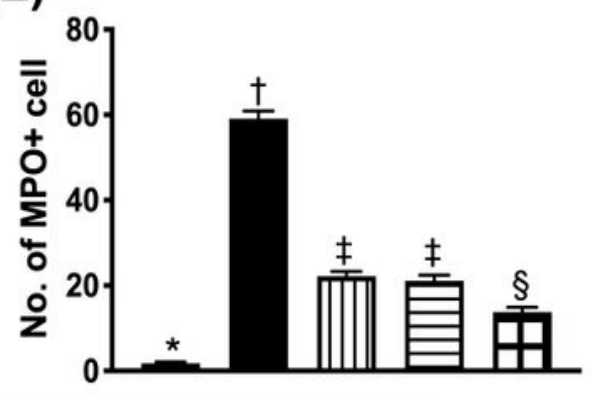
SC TTIR TTIR+Mel TTIR+ADMSC

Figure 7 
Mel-ADMSCs therapy effectively inhibited the cellular level of inflammatory reaction in testis by $72 \mathrm{~h}$ after IR procedure A to E) Illustrating the microscopic finding (400x) of immunohistochemical (IHC) stain for identification of positively stained matrix metalloproteinase (MMP-9) cells (gray color). F) Analytical result of number of MMP-9+ cells, * vs. other groups with different symbols $(\dagger, \neq, \S), p<0.0001$. G to $K)$ Illustrating the microscopic finding (400x) of IHC stain for identification of positively stained myeloperoxidase (MPO) cells (gray color). F) Analytical result of number of MPO+ cells, * vs. other groups with different symbols $(t, \neq, \S), p<0.0001$. All statistical analyses were performed by one-way ANOVA, followed by Bonferroni multiple comparison post hoc test ( $n=6$ for each group). Symbols $\left({ }^{*}, t, \neq, \S\right)$ indicate significance (at 0.05 level). SC = sham-operated control; TTIR = testicular torsion-induced ischemia-reperfusion; $\mathrm{Mel}=$ melatonin; $\mathrm{ADMSCs}$ = adipose-derived mesenchymal stem cells. 